\title{
CONTACT GEOMETRY AND CR-STRUCTURES ON SPHERES
}

\author{
JOHN BLAND \\ Department of Mathematics, University of Toronto \\ Toronto, Ontario M5S 1A1, Canada \\ E-mail: bland@math.toronto.edu \\ TOM DUCHAMP \\ Department of Mathematics, University of Washington GN-50 \\ Seattle, Washington 98195, U.S.A. \\ E-mail: duchamp@math.washington.edu
}

\begin{abstract}
A normal form for small CR-deformations of the standard CR-structure on the $(2 n+1)$-sphere is presented. The space of normal forms is parameterized by a single function on the sphere. For $n>1$, the normal form is used to obtain explicit embeddings into $\mathbb{C}^{n+1}$. For $n=1$, the cohomological obstruction to embeddability is identified.
\end{abstract}

1. Introduction. In this paper, we study the space of strongly pseudoconvex CR-structures on the sphere $S^{2 n+1}$ in a neighborhood of the standard CRstructure given by the standard embedding as the unit sphere in $\mathbb{C}^{n+1}$. Three questions present themselves:

(1) Which CR-structures near the standard one arise as the boundaries of domains in $\mathbb{C}^{n+1}$ ? (Such CR-structures are said to be embeddable.)

(2) Is there a normal form for CR-structures near the standard one?

(3) Is there a natural parameterization of the space of equivalence classes of CR-structures, where two CR-structures near the standard one are said to

1991 Mathematics Subject Classification: Primary 32F40; Secondary 32G07, 32F15.

Key words and phrases: CR-structures, convex domains, contact geometry, deformation theory, moduli, Riemann maps.

Partially supported by an NSERC grant.

The second author wishes to thank the University of Toronto, where portions of this paper were written.

The paper is in final form and no version of it will be published elsewhere. 
be equivalent if they are CR-isomorphic via a diffeomorphism which is near the identity.

There is a dichotomy between the cases $n>1$ and $n=1$. Results of Boutet de Monvel [BdM] show that, when $n$ is greater than 1 , all CR-structures near the standard one are embeddable; and, as we will see here, the results of Lempert [L1] and Bland-Duchamp [BD1] give a normal form.

In the case $n=1$, an example of Rossi $[\mathrm{R}]$ shows that there exist CR-structures near the standard one which are not embeddable. In [BE], Burns and Epstein showed that they are almost never embeddable. A recent result of Lempert [L2] showed that if the CR-structure is sufficiently near the standard one and embeddable in $\mathbb{C}^{m}$ for some $m$, then there is an embedding near the standard sphere. This fact was used in [B] to show that the embeddable CR-structures form a Hilbert submanifold in the space of all CR-structures and to give a normal form. Cheng and Lee [CL] have proved the existence of a local slice for the action of the contact diffeomorphisms on the space of CR-structures. Our goal here is to outline a framework within which these results naturally fit. Most of the technical machinery needed is contained in [BD1], [B]. Details will appear in [BD2].

\section{Abstract CR-structures on spheres}

2.1. Basic facts. We begin with a review of some basic facts about CRstructures.

Definition 2.1. An n-dimensional Cauchy-Riemann structure (CR-structure) on $S^{2 n+1}$ is a rank $n$ complex subbundle $H_{(1,0)} \subset T_{\mathbb{C}} S^{2 n+1}$ of the complexified tangent bundle of $S^{2 n+1}$ such that

(1) $H_{(1,0)} \cap H_{(0,1)}=0$,

(2) $H_{\mathbb{C}}=H_{(1,0)} \bigoplus H_{(0,1)} \subset T_{\mathbb{C}} S^{2 n+1}$ has complex codimension one, where, as usual, $H_{(0,1)}$ denotes the conjugate bundle $\overline{H_{(1,0)}}$,

(3) the integrability condition $[\bar{X}, \bar{Y}] \in \Gamma\left(H_{(0,1)}\right)$ is satisfied for all smooth sections $\bar{X}, \bar{Y} \in \Gamma\left(H_{(0,1)}\right)$.

The bundle $H_{(1,0)}$ is called the holomorphic tangent bundle of the CR-structure.

Two CR-structures $H_{(1,0)}$ and $\hat{H}_{(1,0)}$ are said to be equivalent if there is a diffeomorphism $F: S^{2 n+1} \rightarrow S^{2 n+1}$ such that $F_{*} H_{(1,0)}=\hat{H}_{(1,0)}$. We are only interested in CR-structures up to equivalence. Observe that $H_{\mathbb{C}}$ is the complexification of a real codimension one subbundle $H \subset T S^{2 n+1}$ consisting of vectors of the form $X+\bar{X}, X \in H_{(1,0)}$. Let $\eta$ be a 1 -form dual to $H$. The CR-structure $H_{(1,0)}$ is said to be strongly pseudoconvex if $-i d \eta(X, \bar{X})>0$ for all non-zero $X \in H_{(1,0)}$. In this case, $\eta \wedge(d \eta)^{n}$ is a nowhere vanishing $(2 n+1)$-form. A real 1-form $\eta$ satisfying this latter condition is called a contact form and $H$ is called a contact distribution. A diffeomorphism leaving the contact distribution fixed is called a contact diffeomorphism. 
The most important examples of CR-structures are those arising from domains in $\mathbb{C}^{n+1}$. Let $\rho$ be a smooth nonnegative function on $\mathbb{C}^{n+1}$ and let $D=\left\{z \in \mathbb{C}^{n+1}\right.$ : $\rho(z)<1\}$ be a bounded domain. The boundary $\partial D$ is a CR-manifold for which the holomorphic tangent bundle is the intersection of the complexified tangent bundle of $\partial D$ with the holomorphic tangent bundle of $\mathbb{C}^{n+1}$, and, if the pullback to $\partial D$ of the 1 -form $i \bar{\partial} \rho$ is a contact form, then it is strongly pseudoconvex $\left(^{1}\right)$.

The standard $C R$-structure is the one induced by the standard embedding $S^{2 n+1} \subset \mathbb{C}^{n+1}$, and the standard contact form is the restriction of $i \bar{\partial}|z|^{2}$ to $S^{2 n+1}$, where $\left(z^{1}, \ldots, z^{n+1}\right)$ are the coordinates for $\mathbb{C}^{n+1}$. Henceforth, $H_{(1,0)} \subset T_{\mathbb{C}} S^{2 n+1}$ denotes the holomorphic tangent bundle of the standard CR-structure, $\eta$ denotes the standard contact form, and $H_{\mathbb{C}}$ denotes the complexification of the standard contact distribution. Objects associated to any other CR-structure will be decorated with hats. The symbol Diff $_{H}$ denotes the infinite-dimensional group of orientation preserving contact diffeomorphisms of $S^{2 n+1}$.

Two strongly pseudoconvex CR-structures on $S^{2 n+1}$ are said to be isotopic if they can be connected by a smooth 1-parameter family of strongly pseudoconvex CR-structures. In this paper, we consider only strongly pseudoconvex CR-structures which are isotopic to the standard one on $S^{2 n+1}$.

2.2. Representation by deformation tensors. Every CR-structure which is isotopic to the standard one can be represented by a deformation tensor.

The proof of this fact relies on a theorem of John Gray $[G]$ which states that all contact structures on a compact manifold near a fixed contact structure are equivalent:

THEOREM 2.1 (Gray). Let $\eta_{t}$ be a differentiable family of contact structures on a compact $2 n+1$ dimensional manifold $M$. Then there is a differentiable family of diffeomorphisms $F_{t}: M \mapsto M$ and a family of non-vanishing functions $p_{t}$ such that

$$
F_{t}^{*}\left(\eta_{t}\right)=p_{t} \eta_{0}
$$

Corollary 2.1. Every strongly pseudoconvex CR-structure on $S^{2 n+1}$ which is isotopic to the standard one is CR-equivalent to one of the form

$$
\hat{H}_{(1,0)}=\left\{X-\bar{\phi}(X): X \in H_{(1,0)}\right\}
$$

where $\phi: H_{(0,1)} \rightarrow H_{(1,0)}$ a complex vector bundle map, called the deformation tensor for $\hat{H}_{(1,0)}$.

P r o of. That the CR-structure is equivalent to one satisfying the inclusion relation $\hat{H}_{(1,0)} \subset H_{\mathbb{C}}$ is clear from Gray's theorem. Thus, there is a family $\hat{H}_{(1,0)}(t)$, $t \in[0,1]$, joining $H_{(1,0)}$ to $\hat{H}_{(1,0)}$. For $t$ small, it is clear that there are bundle maps $\phi(t)$ such that $\hat{H}_{(1,0)}(t)$ is the graph of $-\bar{\phi}(t)$. The integrability conditions

$\left({ }^{1}\right)$ The fact that $D$ is bounded forces the Levy form to be positive everywhere on $\partial D$. 
for CR-structures (see below) imply that $\phi(t)$ satisfies certain symmetry properties and an a priori bound on the size of $\phi(t)$, from which the result follows. (See [BD1, page 83] where a similar argument is given.)

2.3. Geometry of the standard CR-structure. There is a natural circular action on the sphere which leaves the standard CR-structure invariant-namely, multiplication by the group $U(1)$ of unimodular complex scalars. Let $T$ be the generator of this $U(1)$-action with period $2 \pi$. The vector field $T$ is characterized by the two conditions

$$
T\lrcorner \eta=1, \quad T\lrcorner d \eta=0 .
$$

The orbit space of this action is the set of complex lines through the origin, $\mathbb{C} P^{n}$, and the quotient map is the Hopf fibration.

Often, it will be worthwhile to do calculations using local coordinates. We will fix our notation now. Let $\left(w^{1}, \ldots, w^{n}, \theta\right)$ be local coordinates for $S^{2 n+1}$, where $\left(w^{1}, \ldots, w^{n}\right)$ are the local inhomogeneous coordinates for $\mathbb{C} P^{n}$ defined by the equations $w^{j}=z^{j} / z^{n+1}$, and $\theta$ is a local fiber coordinate; that is, the $w$ variables parametrize the complex lines through the origin, and $\theta$ determines a point on the line at unit distance from the origin of $\mathbb{C}^{n+1}$. Define the function $u:=\log \left(1+|w|^{2}\right)$. In these local coordinates,

$$
\begin{aligned}
T & =\frac{\partial}{\partial \theta}, \\
\eta & =d \theta+\operatorname{Re}(i \bar{\partial} u)=d \theta+\frac{i}{2}\left(u_{\bar{\alpha}} d w^{\bar{\alpha}}-u_{\alpha} d w^{\alpha}\right),
\end{aligned}
$$

where we have used the notation $u_{\alpha}=\partial u / \partial w^{\alpha}$. A local framing for $H_{(1,0)}$ is given by the vector fields

$$
\mathrm{e}_{\alpha}:=\frac{\partial}{\partial w^{\alpha}}+\frac{i}{2} u_{\alpha} T
$$

and the dual coframing is given by the 1 -forms $d w^{\alpha}, 1 \leq \alpha \leq n$.

Notice that $\eta$ defines a connection form on the circle bundle over $\mathbb{C} P^{n}$, that $\mathrm{e}_{\alpha}$ is the horizontal lift of the vector field $\partial / \partial w^{\alpha}$, and that

$$
d \eta=i u_{\alpha \bar{\beta}} d w^{\alpha} \wedge d w^{\bar{\beta}}
$$

is a nondegenerate 2 form. The form

$$
\Omega:=-i d \eta=\partial \bar{\partial} u=u_{\alpha \bar{\beta}} d w^{\alpha} \wedge d w^{\bar{\beta}}
$$

is the Fubini Study metric on $\mathbb{C} P^{n}$.

2.4. Integrability conditions. Consider now a CR-structure of the form (1). In local coordinates,

$$
\phi=\phi_{\bar{\beta}}{ }^{\alpha} d w^{\bar{\beta}} \otimes \mathrm{e}_{\alpha}
$$

and $\hat{H}_{(0,1)}$ is spanned locally by the vector fields

$$
\hat{e}_{\bar{\alpha}}:=\mathrm{e}_{\bar{\alpha}}-\phi{ }_{\bar{\alpha}}^{\beta} \mathrm{e}_{\beta} .
$$


Expansion of the brackets $\left[\hat{e}_{\bar{\alpha}}, \hat{e}_{\bar{\beta}}\right]$ using the commutation relations

$$
\left[T, \mathrm{e}_{\alpha}\right]=0, \quad\left[T, \mathrm{e}_{\bar{\alpha}}\right]=0, \quad\left[\mathrm{e}_{\alpha}, \mathrm{e}_{\beta}\right]=0, \quad\left[\mathrm{e}_{\alpha}, \mathrm{e}_{\bar{\beta}}\right]=-i u_{\alpha \bar{\beta}} T
$$

immediately yields the conditions:

(A) (Symmetry): $\phi_{\bar{\alpha} \bar{\beta}}=\phi_{\bar{\beta} \bar{\alpha}}$, where $\phi_{\bar{\alpha} \bar{\beta}}=: \phi_{\bar{\alpha}}^{\gamma} u_{\gamma \bar{\beta}}$.

(B) (Horizontal integrability): $\bar{\partial}_{b} \phi-\frac{1}{2}[\phi, \phi]=0$, where

$$
\bar{\partial}_{b} \phi=\frac{\partial \phi_{\bar{\beta}}^{\gamma}}{\partial w^{\bar{\alpha}}} d w^{\bar{\alpha}} \wedge d w^{\bar{\beta}} \otimes \mathrm{e}_{\gamma}
$$

and where $[$,$] is the bracket operation$

$$
[\phi, \phi]=d w^{\bar{\alpha}} \wedge d w^{\bar{\beta}} \otimes\left[\phi_{\bar{\alpha}}{ }^{\delta} \mathrm{e}_{\delta}, \phi_{\bar{\beta}}{ }^{\sigma} \mathrm{e}_{\sigma}\right]
$$

Recall that we assumed $\hat{H}_{(1,0)}$ to be isotopic to the standard structure. There is, therefore, a family of tensors $\phi(t)$ with $\phi(0)=0$ and $\phi(1)=\phi$. The condition $\hat{H}_{(1,0)} \cap \hat{H}_{(0,1)}=0$ shows that the composition

$$
\phi(t) \circ \bar{\phi}(t): H_{(1,0)} \rightarrow H_{(1,0)}
$$

cannot have eigenvalue one. On the other hand, the symmetry condition can be used to show that all eigenvalues are nonnegative real numbers. Hence, all eigenvalues are bounded above by 1 . This is equivalent to the following condition:

(C) (Nondegeneracy): $\left|\phi_{\alpha \beta} X^{\alpha} X^{\beta}\right|<u_{\alpha \bar{\beta}} X^{\alpha} X^{\bar{\beta}}$ for all $X \in \mathbb{C}^{n}$.

Let $\mathcal{D}_{n}$ denote the space of deformation tensors satisfying conditions $(\mathrm{A}),(\mathrm{B})$ and (C). Every strongly pseudoconvex CR-structure on $S^{2 n+1}$ which is isotopic to the standard one is equivalent to one defined by an element of $\mathcal{D}_{n}$. Moreover, the group of contact diffeomorphisms of $S^{2 n+1}$ acts on the set of such tensors in a natural way. Consequently, the classification of these CR-structures on $S^{2 n+1}$ can be achieved by the two-step program of (1) studying the space $\mathcal{D}_{n}$ and (2) studying the action of the group of contact diffeomorphisms on it.

3. The space $\mathcal{D}_{n}$. Our analysis of the space $\mathcal{D}_{n}$ mimics the approach to deformation theory of complex structures as developed by Kuranishi. We construct a complex of certain vector-valued forms. Deformation tensors are the 1-forms of the complex which satisfy a nonlinear differential equation (the integrability condition $(\mathrm{B})$ ), and these are parameterized by a certain component of their harmonic decomposition.

3.1. The symmetric complex $\left(\Omega_{\sigma}^{(0, \bullet)}\left(H_{(1,0)}\right), \bar{\partial}_{b}\right)$. We will work on a subcomplex of the $\bar{\partial}_{b}$-complex of Kohn-Rossi. The symbols $\Omega^{(0, q)}$ and $\Omega^{(0, q)}\left(H_{(1,0)}\right)$ denote the spaces of smooth scalar and $H_{(1,0)}$-valued forms on $S^{2 n+1}$. Thus, elements of $\Omega^{(0, q)}$ and $\Omega^{(0, q)}\left(H_{(1,0)}\right)$ are forms of the types

$$
\tau=\tau_{\bar{B}} d w^{\bar{B}} \text { and } \quad \tau=\tau_{\bar{B}}^{\alpha} d w^{\bar{B}} \otimes \mathrm{e}_{\alpha},
$$


respectively. (The standard summation and multi-index conventions are in force throughout.) The operator $\bar{\partial}_{b}$ is given by the formula

$$
\bar{\partial}_{b} \tau=\mathrm{e}_{\bar{\beta}}\left(\tau_{\bar{B}}{ }^{\alpha}\right) d w^{\bar{\beta}} \wedge d w^{\bar{B}} \otimes \mathrm{e}_{\alpha} .
$$

In order to take into account the symmetry condition (A) above, we introduce the hook product of $\tau$ and $\Omega$, written $\tau \bar{\wedge} \Omega$ :

$$
\tau \bar{\wedge} \Omega=\tau_{\bar{B}}{ }^{\alpha} u_{\alpha \bar{\gamma}} d w^{\bar{B}} \wedge d w^{\bar{\gamma}} .
$$

The hook product operation is the process of lowering one index via the Hermitian metric $u_{\alpha \bar{\beta}} d w^{\alpha} \otimes d w^{\bar{\beta}}$ followed by skew-symmetrization. In the special case of vector fields (vector forms of type $(0,0)$ ), the hook product is just interior evaluation. Observe that a tensor $\phi \in \Omega^{(0,1)}\left(H_{(1,0)}\right)$ satisfies condition (A) precisely when

$$
\phi \pi \Omega=0 .
$$

The identity $\phi \pi \Omega=0$ generalizes, for $q>0$, to define the subcomplex of symmetric vector-valued forms:

$$
\Omega_{\sigma}^{(0, q)}\left(H_{(1,0)}\right)=\{\tau: \tau \pi \Omega=0\} .
$$

For $q=0$ this definition will not suffice, since $X \bar{\wedge} \Omega=0$ for $X \in H_{(1,0)}$ implies that $X=0$; we proceed somewhat differently. We need a partial inverse of the hook product, written ( $)^{\#}$. Let $\eta=\eta_{\bar{B}} d w^{\bar{B}}$ be a horizontal $(0, q)$-form. Then $\eta^{\#}$ is the vector valued $(0, q-1)$-form defined by the local coordinate formula,

$$
\eta^{\#}=\eta_{\bar{\beta}_{1} \ldots \bar{\beta}_{q-1} \bar{\gamma}} c^{\alpha \bar{\gamma}} d w^{\bar{\beta}_{1}} \wedge \ldots \wedge d w^{\bar{\beta}_{q-1}} \otimes \mathrm{e}_{\alpha},
$$

where $\left(u^{\alpha \bar{\beta}}\right)$ is the inverse of the matrix $\left(u_{\alpha \bar{\beta}}\right)$.

The requirement that $\bar{\partial}_{b} X$ be an element of $\Omega_{\sigma}^{(0,1)}\left(H_{(1,0)}\right)$ forces the identity $\bar{\partial}_{b} X \bar{\wedge} \Omega=0$. A simple local calculation then shows that (locally) such a vector field must be of the form

$$
X=\left(\bar{\partial}_{b} f\right)^{\#}
$$

for $f$ a complex-valued function on $S^{2 n+1}$. We define the space of symmetric 0 -forms by the equation

$$
\Omega_{\sigma}^{(0,0)}\left(H_{(1,0)}\right)=\left\{X \in \Omega^{(0,0)}\left(H_{(1,0)}\right): X=\left(\bar{\partial}_{b} f\right)^{\#}, f \in C^{\infty}\right\},
$$

where $C^{\infty}$ denotes the space of smooth, complex-valued functions on $S^{2 n+1}$. We call $\Omega_{\sigma}^{(0,0)}\left(H_{(1,0)}\right)$ the space of horizontal Hamiltonian vector fields.

Definition 3.1. The symmetric complex $\left(\Omega_{\sigma}^{(0, \bullet)}\left(H_{(1,0)}\right), \bar{\partial}_{b}\right)$ is the differential complex

$$
0 \rightarrow \Omega_{\sigma}^{(0,0)}\left(H_{(1,0)}\right) \stackrel{\bar{\partial}_{b}}{\longrightarrow} \Omega_{\sigma}^{(0,1)}\left(H_{(1,0)}\right) \stackrel{\bar{\partial}_{b}}{\longrightarrow} \Omega_{\sigma}^{(0,2)}\left(H_{(1,0)}\right) \stackrel{\bar{\partial}_{b}}{\longrightarrow} \ldots
$$

R e m a r k. The complex Hamiltonian vector fields just defined are related to contact vector fields. Recall that a contact vector field is a real vector field $Y$ 
satisfying the condition

$$
\mathcal{L}_{Y} \eta=h \eta,
$$

for some function $h$ on $S^{2 n+1}$. When $f=g i$, with $g$ real, the complex Hamiltonian vector field $X=\left(\bar{\partial}_{b} f\right)^{\#}$ is the $(1,0)$ part of a contact vector field. More precisely,

$$
X_{f}=-i f T+X+\bar{X}
$$

is a contact vector field. Notice that $\left.f=X_{f}\right\lrcorner i \eta$, and $\mathcal{L}_{X_{f}} \eta=-i T(f) \eta$.

Using the Folland-Stein estimates, it is possible to develop a Hodge theory for the symmetric complex with good estimates on the orthogonal complement of the harmonic forms. Thus, there are homotopy maps $h_{\sigma}: \Omega_{\sigma}^{(0, q)}\left(H_{(1,0)}\right) \rightarrow$ $\Omega_{\sigma}^{(0, q-1)}\left(H_{(1,0)}\right)$ which give rise to the symmetric decomposition

$$
\tau=\bar{\partial}_{b} \circ h_{\sigma}(\tau) \oplus h_{\sigma} \circ \bar{\partial}_{b}(\tau) \oplus H_{\sigma}(\tau),
$$

where $H_{\sigma}: \Omega_{\sigma}^{(0, q)}\left(H_{(1,0)}\right) \rightarrow H^{q}\left(\Omega_{\sigma}^{(0, \bullet)}\left(H_{(1,0)}\right), \bar{\partial}_{b}\right)$ is orthogonal projection onto the harmonic symmetric forms. In degree 0 , the symmetric decomposition assumes the form

$$
X=h_{\sigma} \circ \bar{\partial}_{b}(X) \oplus H_{\sigma}(X) .
$$

Let $\Omega_{\sigma, s}^{(0, q)}\left(H_{(1,0)}\right)$ and $\Gamma^{s}$ be the Folland-Stein completions of $\Omega_{\sigma}^{(0, q)}\left(H_{(1,0)}\right)$ and $C^{\infty}$, with respect to the Folland-Stein norm \|\|$_{s}$ (see [BD1]). Let $\mathcal{D}_{n, s}$ denote the Folland-Stein completion of the space $\mathcal{D}_{n}$. In [BD2] we prove the following theorem.

TheOREM 3.1. Suppose $n>1$ and $s>2 n+4$. Then $H^{1}\left(\Omega_{\sigma}^{(0, \bullet)}\left(H_{(1,0)}\right), \bar{\partial}_{b}\right)=0$ and $\mathcal{D}_{n, s}$ is a smooth Hilbert manifold.

Moreover, let

$$
\mathcal{N}_{\epsilon}=\left\{f \in \Gamma^{s+2}: f \perp \operatorname{ker}\left(\bar{\partial}_{b} \circ\left(\bar{\partial}_{b}(\cdot)\right)^{\#}\right),\|f\|_{s+2}<\epsilon\right\}
$$

and assume that $\epsilon$ is sufficiently small. Then $\mathcal{N}_{\epsilon}$ is diffeomorphic to a neighborhood $\mathcal{N}^{\prime} \subset \mathcal{D}_{n, s}$. More precisely, every deformation tensor $\phi \in \mathcal{N}^{\prime}$ can be uniquely expressed in the form

$$
\phi=\bar{\partial}_{b}\left(\bar{\partial}_{b} f_{\phi}\right)^{\#} \oplus h_{\sigma} \bar{\partial}_{b} \phi
$$

which defines a diffeomorphism $\phi \mapsto f_{\phi}$ from $\mathcal{N}^{\prime}$ to $\mathcal{N}_{\epsilon}$.

The function $f_{\phi}$ introduced in Theorem 3.1 is called the generating function for the deformation tensor $\phi$.

4. Normal form $(n>1)$. The parameterization in Theorem 3.1 is not optimal. Since the action of the group of contact diffeomorphisms on deformation tensors has not been taken into account, each equivalence class of CR-structures is represented by an infinite dimensional family of deformation tensors. In this section, we show how to normalize small deformation tensors via this action by contact diffeomorphisms. 
4.1. The Fourier decomposition. The $U(1)$-action on $S^{2 n+1}$ preserves the standard CR-structure, as well as the contact form $\eta$. It, therefore, induces an action on the $\bar{\partial}_{b}$-complex, which is equivariant with respect to the operators $\bar{\wedge},($ ) $\#$ and $\bar{\partial}_{b}$. The direct sum decomposition of the spaces $\Omega^{(0, q)}\left(H_{(1,0)}\right)$ into eigenspaces of the $U(1)$-action is called the Fourier decomposition. Thus, each vector form $\tau \in \Omega^{(0, q)}\left(H_{(1,0)}\right)$ has the decomposition

$$
\tau=\sum_{k=-\infty}^{+\infty} \tau_{k} \quad \text { where } \quad \tau_{k}=\tau_{k, \bar{B}}{ }^{\alpha} e^{i k \theta} d w^{\bar{B}} \otimes \mathrm{e}_{\alpha} .
$$

and $\tau_{k, \bar{B}}{ }^{\alpha}$ is independent of $\theta$. If $\tau$ is symmetric, then so are each of the terms in its Fourier decomposition. The decomposition of tensors into positive, negative and zeroth order Fourier components gives the splitting

$$
\Omega_{\sigma}^{(0, q)}\left(H_{(1,0)}\right)=\Omega_{\sigma,-}^{(0, q)}\left(H_{(1,0)}\right) \oplus \Omega_{\sigma, 0}^{(0, q)}\left(H_{(1,0)}\right) \oplus \Omega_{\sigma,+}^{(0, q)}\left(H_{(1,0)}\right)
$$

and we write $\tau=\tau_{-}+\tau_{0}+\tau_{+}$. Similar decompositions are defined for ordinary forms and for functions. If $f$ is a real-valued function on $S^{2 n+1}$ then its Fourier decomposition

$$
f=f_{-}+f_{0}+f_{+}
$$

satisfies the identities $f_{+}=\overline{f_{-}}$and, consequently, $f_{-}=\overline{f_{+}}$.

Of particular interest are the tensors with only positive Fourier components. Such tensors extend holomorphically along each of the disks of the unit disk bundle of $E \rightarrow \mathbb{C} P^{n}$, and, because they vanish on the zero section, they descend to the blow-down - to tensors on the unit ball in $\mathbb{C}^{n+1}$. Tensors with only a zeroth Fourier component are pull-backs to $E$ of forms on $\mathbb{C} P^{n}$, and are called basic. (Here we are implicitly using the fact that the holomorphic tangent bundle of $S^{2 n+1}$ is the horizontal lift of the holomorphic tangent bundle of $\mathbb{C} P^{n}$ via the connection form $\eta$.)

4.2. Action of the contact diffeomorphisms. We now consider the action of the group of contact diffeomorphisms on the space of deformation tensors. This action is not strictly a pullback of the deformation tensor; rather, it pulls back the CR structure, and considers the new CR structure as a deformation of the standard one. More specifically, we have the following definition.

Definition 4.1. Let $\phi$ be a deformation tensor on $S^{2 n+1}$ and $F: S^{2 n+1} \rightarrow$ $S^{2 n+1}$ a contact diffeomorphism and let $\hat{H}_{(1,0)} \subset H_{\mathbb{C}}$ be the bundle of vectors of type $(1,0)$ defined by $\phi$. There a unique subbundle $\hat{H}_{(1,0)}^{\prime} \subset H_{\mathbb{C}}$ such that the equation $F_{*} \hat{H}_{(1,0)}^{\prime}=\hat{H}_{(1,0)}$ holds. The pullback of $\phi$ by $F$, written $F^{*} \phi$, is the deformation tensor whose space of $(1,0)$-vectors is $\hat{H}_{(1,0)}^{\prime}$.

This defines an action

$$
\Psi: \operatorname{Diff}_{H} \times \mathcal{D}_{n} \rightarrow \mathcal{D}_{n}, \quad(F, \phi) \mapsto F^{*} \phi .
$$


To compute the derivative of the map $\Psi$ at the point $(i d, 0)$, we work formally, leaving details to $[\mathrm{B}]$ and [BD2].

Let $F_{t}=i d+t X_{f}+O\left(t^{2}\right)$ denote the one parameter family of contact diffeomorphisms which is generated by a contact vector field $X_{f}$, where $f=g i$ is pure imaginary as above. Let $\phi_{t}=t \phi+O\left(t^{2}\right)$ be a family of deformation tensors and let $X=\left(\bar{\partial}_{b} f\right)^{\#}=X^{\alpha} \mathrm{e}_{\alpha}$. We calculate (to first order in $t$ ) as follows. Recall that the vectors of type $(0,1)$ with respect to $\phi_{t}$ are spanned by $\hat{\mathrm{e}}_{\bar{\beta}}=\mathrm{e}_{\bar{\beta}}-\phi_{\bar{\beta}, t}{ }^{\gamma} \mathrm{e}_{\gamma}$. Then, since $F_{t}^{-1}=i d-t X_{f}+O\left(t^{2}\right)$, the pull-back under $F_{t}$ of the space of $(0,1)$-vectors is spanned by the set

$$
d F_{t}^{-1} \hat{\mathrm{e}}_{\bar{\beta}}=\mathrm{e}_{\bar{\beta}}-t \mathrm{e}_{\bar{\beta}}\left(X^{\gamma}\right) \mathrm{e}_{\gamma}-t \mathrm{e}_{\bar{\beta}}\left(X^{\bar{\gamma}}\right) \mathrm{e}_{\bar{\gamma}}-t \phi_{\bar{\beta}}{ }^{\gamma} \mathrm{e}_{\gamma}+O\left(t^{2}\right) .
$$

After a change of basis, we find that it is also spanned by vectors of the form

$$
\mathrm{e}_{\bar{\beta}}-t\left(\phi_{\bar{\beta}}^{\gamma}+\mathrm{e}_{\bar{\beta}}\left(X^{\gamma}\right)\right) \mathrm{e}_{\gamma}+O\left(t^{2}\right) \text {. }
$$

Thus, the derivative $d \Psi_{(i d, 0)}$ has the form

$$
d \Psi_{(i d, 0)}: T \operatorname{Diff}_{H} \times T \mathcal{D} \rightarrow T \mathcal{D}, \quad\left(X_{f}, \phi\right) \mapsto \phi+\bar{\partial}_{b}\left(\bar{\partial}_{b} f\right)^{\#},
$$

where $f=g i$ for some real-valued function $g$.

4.3. Cancelling negative Fourier coefficients. We now show how the action of the group of contact diffeomorphisms can be used to normalize deformation tensors. As we have just seen, the infinitesimal action is of the form

$$
\phi \mapsto \phi+\bar{\partial}_{b}\left(\bar{\partial}_{b} f\right)^{\#},
$$

where $f$ is pure imaginary. We have also seen that, for $n>1, \phi$ has the decomposition

$$
\phi=\bar{\partial}_{b}\left(\bar{\partial}_{b} f_{\phi}\right)^{\#}+h_{\sigma}\left(\bar{\partial}_{b} \phi\right)
$$

and that $\phi$ is uniquely determined by its generating function $f_{\phi}$.

Notice that, one may construct a pure imaginary function by starting with an arbitrary function with no positive Fourier components and subtracting from it the conjugate of its negative Fourier components. We can use this freedom to modify the negative (and part of the zeroth) Fourier components of a deformation tensor via the action of the group of contact diffeomorphisms. An application of the Implicit Function Theorem for Banach spaces gives the following normal form result, which shows that every sufficiently small deformation of the standard CRstructure on the spheres $S^{2 n+1}, n>1$ is representable by a deformation tensor with no negative Fourier components.

THEOREM 4.1. Let $n>1$ and $s>2 n+4$. Then there is a number $\epsilon>0$ depending on $n$ and $s$ such that each deformation tensor $\phi \in \mathcal{D}_{n, s}$ with $\|\phi\|_{s}<\epsilon$ is CR-equivalent to a deformation tensor in the normal form

$$
\bar{\partial}_{b}\left(\bar{\partial}_{b} f\right)^{\#}+h_{\sigma}(\tau)
$$


where $f \in \Gamma_{s+2}\left(S^{2 n+1}\right)$ and $\tau \in \Omega_{\sigma, s+1}^{(0,2)}\left(H_{(1,0)}\right)$ have Fourier decompositions of the forms

$$
f=\sum_{k=0}^{+\infty} f_{k} \text { and } \tau=\sum_{k=0}^{+\infty} \tau_{k},
$$

where $f_{0}$ is the pull-back of a real-valued function on $\mathbb{C} P^{n}$. The tensor $\tau$ is uniquely determined by the generating function $f$.

Remark. The normal form constructed in this theorem is unique up to specification of a finite number of additional parameters. This is clear from the observation that the linearized mapping $d \Psi_{(i d, 0)}$ has a finite dimensional kernel of the same dimension as the automorphism group of the standard CR structure on the sphere. Specifying these parameters can be interpreted as determining a point on the sphere, and a second order framing at that point.

Remark. The proof of this theorem will appear in [BD2]. It involves endowing the neighborhood of the identity in Diff ${ }_{H}$ with a Hilbert space structure which is compatible with the Folland-Stein norms. With respect to this structure, one can show that the action $\Psi$ is of class $C^{1}$ on the Folland-Stein completions of Diff ${ }_{H}$ and $\mathcal{D}_{n}$.

Omori $[\mathrm{O}]$ has analyzed the action of the contact diffeomorphism group in great generality; our analysis is similar to his. Omori's treatment, however, is based on the standard Sobolev spaces while our work uses the anisotropic norms of Folland-Stein. This fundamental difference means that a much more detailed analysis is required. This analysis, in the case of $S^{3}$, is contained in [B], and the higher dimensional cases will be discussed in [BD2]

4.4. Recovering the indicatrix. In general, it is impossible to eliminate the zeroth Fourier component of a deformation tensor through the action of the contact diffeomorphism group. For suppose that we have normalized to a deformation tensor of the form $\phi=\sum_{k=0}^{\infty} \phi_{k}$, where $\phi$ is a small deformation with $\phi_{0} \neq 0$. Since $\phi_{0}$ descends to define a deformation of the complex structure $\mathbb{C} P^{n}$, and $\mathbb{C} P^{n}$ is stable, there is a small diffeomorphism, say $F$, of $\mathbb{C} P^{n}$ which is an biholomorphism between $\mathbb{C} P^{n}$ with the complex structure defined by $\phi_{0}$ and its standard structure (i.e. $\left.F^{*}(0)=\phi_{0}\right)$. Notice that $F$ is unique up to a biholomorphism of $\mathbb{C} P^{n}$ and it is generally not symplectic. Lift $F$ to a bundle map $\tilde{F}: S^{2 n+1} \rightarrow S^{2 n+1}$. Since $F$ does not preserve the symplectic form of $\mathbb{C} P^{n}$ (the curvature form of the connection $\eta$ ), $\tilde{F}$ does not preserve the connection form $\eta$. Let $\eta^{\prime}$ be the unique connection form for which $\tilde{F}^{*} \eta^{\prime}=\eta$. Thus,

$$
\eta^{\prime}=\eta+\beta
$$

where $\beta$ is the pullback of a 1 -form on $\mathbb{C} P^{n}$. Notice that the contact structure on $S^{2 n+1}$ defined by $\eta^{\prime}$ is not the standard one, and it defines a unique (up to a constant dilation) Hermitian norm $h$ on the tautological bundle $E \rightarrow \mathbb{C} P^{n}$. If $S_{h}$ denotes the unit circle bundle with respect to this new norm, then the map $\tilde{F}$ 
may be interpreted as a CR-isomorphism between $S^{2 n+1}$ with the deformed CRstructure given by $\phi_{0}$ and the standard CR-structure on $S_{h}$ which it inherits as hypersurface of $E$ (or, equivalently, of $\mathbb{C}^{n+1}$ ); that is, we have the CR embedding:

$$
\tilde{F}:\left(S^{2 n+1}, \phi_{0}\right) \rightarrow S_{h} \subset \mathbb{C}^{n+1} .
$$

Now, because $\tilde{F}$ is a $U(1)$-equivariant contact diffeomorphism between $S^{2 n+1}$ and $S_{h}$, the CR-structure defined by $\phi$ is transferred via $\tilde{F}$ to a CR-structure on $S_{h}$ of the form

$$
\phi^{\prime}=\sum_{k=1}^{\infty} \phi_{k}^{\prime} .
$$

The deformation tensor $\phi^{\prime}$ is now to be interpreted as a deformation of the circular domain $S_{h}$ and it is precisely the modular data of the type considered by us in [BD1], where we show it to be the modular data for a marked, linearly convex domain in $\mathbb{C}^{n+1}$. (Recall that a marking of a domain $D \subset \mathbb{C}^{n+1}$ consists of a point $p \in D$ together with a complex basis for the complex tangent space $T_{p} D$.)

In [BD1], we show that the data depends only on the biholomorphism class of $D$ and the marking. Moreover, specifying the marking amounts to specifying $(n+1)^{2}+n+1$ parameters. Thus, the pair $\left(S_{h}, \phi^{\prime}\right)$ is uniquely determined up to the specification of a finite number of additional parameters.

Infinitesimal generating functions for indicatrices. Changing the indicatrix has a nice interpretation within the framework of the symmetric complex. Recall that the sphere is the set of points in $\mathbb{C}^{n+1}$ defined by the equation $|z|^{2}=1$. The indicatrix $S_{h}$ above is defined by the equation

$$
h:=|z|^{2} e^{v}=1
$$

where, by definition, $v=\log \left(h /|z|^{2}\right)$, which descends to a function on $\mathbb{C} P^{n}$. The contact structure on $S^{2 n+1}$ defined by $h$ is the 1 -form

$$
\eta^{\prime}=\eta+\frac{i}{2}(\bar{\partial} v-\partial v)
$$

and the map $\tilde{F}$ discussed above is constructed as follows: Set $\eta_{t}=\eta+t \beta$, where $\beta=\frac{i}{2}(\bar{\partial} v-\partial v)$. Then there is a 1-parameter family of diffeomorphisms $\tilde{F}_{t}$ : $S^{2 n+1} \rightarrow S^{2 n+1}$ such that

$$
\tilde{F}_{t}^{*} \eta_{t}=\eta
$$

which is constructed as follows by a variation of Weinstein's proof of the Darboux theorem $[\mathrm{W}]$. (The technique is due to Moser.) Begin by letting $Y_{t}$ be the time dependent vector field characterized by the two conditions

$$
\left.\left.Y_{t}\right\lrcorner \eta_{t}=0, \quad Y_{t}\right\lrcorner d \eta_{t}=-\beta .
$$

(When $v$ is small, such a vector field exists.) Let $\tilde{F}_{t}$ be the 1-parameter family of diffeomorphisms generated by $Y_{t}$. Then,

$$
\mathcal{L}_{Y_{t}} \eta_{t}=-\beta .
$$


Now compute:

$$
\frac{d}{d t}\left(\tilde{F}_{t}^{*} \eta_{t}\right)=\tilde{F}_{t}^{*}\left(\frac{d \eta_{t}}{d t}+\mathcal{L}_{Y_{t}} \eta_{t}\right)=\tilde{F}_{t}^{*}(\beta-\beta)=0 .
$$

This implies that $\tilde{F}_{t}^{*} \eta_{t}$ is independent of $t$. Since $\tilde{F}_{0}=i d$ and $\tilde{F}_{0}^{*} \eta_{0}=\eta$, it follows that $\tilde{F}_{t}^{*} \eta_{t}=\eta$ for all $t$.

Notice that $Y_{t}=\frac{1}{2}\left(X_{t}+\bar{X}_{t}\right)$ where $X_{t}$ is the $(1,0)$-vector field defined by the equation

$$
\left.X_{t}\right\lrcorner \Omega_{t}=-\bar{\partial} v
$$

where $\Omega_{t}=-i d \eta_{t}$. The lift of $X_{t}$ to a horizontal vector field on $S^{2 n+1}$ is $-\left(\bar{\partial}_{b} v\right)^{\# t}$ and one finds that (to first order)

$$
\phi_{t}:=\tilde{F}_{t}^{*}(0)=-t \bar{\partial}_{b}\left(\bar{\partial}_{b} v\right)^{\#}+O\left(t^{2}\right) .
$$

Thus, the process of transferring to a new indicatrix is represented infinitesimally by a term of the form $-\bar{\partial}_{b}\left(\bar{\partial}_{b} v\right)^{\#}$, for $v$ a real-valued function on $\mathbb{C} P^{n}$.

4.5. Explicit construction of embeddings. When the deformation tensor $\phi$ has sufficiently small Folland-Stein norm $\|\phi\|_{s}, s>2 n+4$, it is possible to obtain explicit embeddings which realize the abstract CR-structure defined by $\phi$ as the CR-structure on a hypersurface. Moreover, when $\phi$ is in normal form, this embedding coincides with a circular map constructed by Lempert [L1] and yields a solution of the homogeneous Monge-Ampére equation on the interior.

An embedding into $\mathbb{C}^{n+1}$ is obtained by constructing $(n+1)$ independent functions which are CR with respect to the deformed CR-structure given by $\phi$. Let $\hat{h}$ be one such function. It is easy to show that a complex-valued function $\hat{h}$ is a CR-function on $S^{2 n+1}$ with respect to the CR-structure defined by $\phi$ if and only if it satisfies the equation

$$
\left(\bar{\partial}_{b}-\phi \cdot \partial_{b}\right) \hat{h}=0
$$

where $\left.\phi \cdot \partial_{b}:=\phi\right\lrcorner \partial_{b}:=\phi_{\bar{\beta}}{ }^{\alpha} \mathrm{e}_{a}$.

To find $\hat{h}$ we write it in the form

$$
\hat{h}=h+g
$$

where $h$ is the harmonic projection of $\hat{h}$ onto the space of CR-functions on $S^{2 n+1}$ with respect to the standard structure. Notice that the function $g=\hat{h}-h$ is $L^{2}$-orthogonal to these CR-functions.

The function $\hat{h}$ can be viewed as a perturbation of $h$. We will show that when the Folland-Stein norm $\|\phi\|_{s}$ is sufficiently small, $h$ determines $g$, and, therefore, it determines $\hat{h}$. To see this, note that from equation (2), it follows that

$$
G \bar{\partial}_{b}^{*}\left(\bar{\partial}_{b}-\phi \cdot \partial_{b}\right) g=G \bar{\partial}_{b}^{*}\left(\phi \cdot \partial_{b} h\right)
$$

Since $g$ is perpendicular to the space of CR-functions, $G \bar{\partial}_{b}^{*} \bar{\partial}_{b} g=g=I g$. Hence, $g$ is a solution of the equation

$$
\left(I-G \bar{\partial}_{b}^{*} \circ \phi \cdot \partial_{b}\right) g=G \bar{\partial}_{b}^{*}\left(\phi \cdot \partial_{b} h\right) .
$$


The operator on the left hand side of this equation is invertible if the operator norm of $G \bar{\partial}_{b}^{*} \circ \phi \cdot \partial_{b}$ is less than one, and this is the case, provided that the Folland-Stein norm $\|\phi\|_{s}$ is sufficiently small. Assume that this is the case and let $g$ be the solution to equation (3).

It remains to show that $\hat{h}$ is a solution of equation (2). To this end, set $\beta=$ $\left(\bar{\partial}_{b}-\phi \cdot \partial_{b}\right) \hat{h}$ and notice that, by construction, $G \bar{\partial}_{b}^{*} \beta=0$. For $n>1$, the $(0,1)$-form $\beta$ has the harmonic decomposition

$$
\beta=\bar{\partial}_{b} G \bar{\partial}_{b}^{*} \beta+G \bar{\partial}_{b}^{*} \bar{\partial}_{b} \beta .
$$

Since $G \bar{\partial}_{b}^{*} \beta=0$, we may compute as follows:

$$
\begin{aligned}
\beta & =G \bar{\partial}_{b}^{*} \bar{\partial}_{b} \beta=G \bar{\partial}_{b}^{*}\left(\bar{\partial}_{b}-\phi \cdot \partial_{b}\right) \beta+G \bar{\partial}_{b}^{*}\left(\phi \cdot \partial_{b} \beta\right) \\
& =G \bar{\partial}_{b}^{*}\left(\bar{\partial}_{b}-\phi \cdot \partial_{b}\right)\left(\bar{\partial}_{b}-\phi \cdot \partial_{b}\right) \hat{h}+G \bar{\partial}_{b}^{*}\left(\phi \cdot \partial_{b} \beta\right)=G \bar{\partial}_{b}^{*}\left(\phi \cdot \partial_{b} \beta\right),
\end{aligned}
$$

where we have used the integrability condition for $\phi$ at the last step. On the other hand, if $\|\phi\|_{s}$ is sufficiently small, then

$$
\|\beta\|_{s+1}=\left\|G \bar{\partial}_{b}^{*}\left(\phi \cdot \partial_{b} \beta\right)\right\|_{s+1}<\|\beta\|_{s+1},
$$

which implies that $\beta=0$.

To obtain an explicit embedding, let $h^{j}, j=1,2 \ldots, n+1$ be the restrictions to $S^{2 n+1}$ of the standard coordinate functions on $\mathbb{C}^{n+1}$, let $\hat{h}^{j}=h^{j}+g^{j}$ be the CR-functions obtained by the procedure we just outlined. They define a map

$$
F: S^{2 n+1} \rightarrow \mathbb{C}^{n+1},
$$

which, by construction, is a CR-map of $\left(S^{2 n+1}, \phi\right)$. By the Sobolev embedding theorem of Folland-Stein [FS], for Folland-Stein norm $\|\phi\|_{s}$ sufficiently small, $s>2 n+4, F$ will be at least $C^{2+\alpha}$ for some $\alpha>0$. Let $D \subset \mathbb{C}^{n+1}$ be the domain bounded by $F\left(S^{2 n+1}\right)$ and note that, for $\phi$ sufficiently small, $D$ is strongly convex.

Recall that, when $\phi$ is in normal form, it has no negative Fourier components. This important fact means that $\phi$ extends holomorphically along the unit disks of the Hermitian vector bundle $E \rightarrow \mathbb{C} P^{n}$ to define an integrable complex structure on the unit disk bundle of $E$ with respect to the standard metric, and that the map $F$ extends to a map which is holomorphic relative to this deformed complex structure. If we transfer the map to the indicatrix $S_{h}$ defined by the zeroth Fourier component of $\phi$, then one can show that the map $F$ descends to a $C^{1}$-map from the indicatrix $I=\left\{z \in \mathbb{C}^{n+1}: h(z) \leq 1\right\}$ into the strongly convex domain $D$. The results of [BD1] show that the map $F$ is one of the circular representations of the strongly convex domain $D$ constructed by Lempert [L1]. We have, thus, recovered Lempert's construction for domains in a neighborhood of the round sphere.

5. Normal form $n=1$. The case of $S^{3}$ is both simpler and more subtle than the higher dimensional case $S^{2 n+1}, n>1$. It is simpler because both the symmetry condition (A) and the integrability condition (B) are vacuous - there 
are no 2-forms - and because there is a global framing of the holomorphic tangent bundle $H_{(1,0)}$ which facilitates computations. It is more subtle because the degree 1 cohomology of the symmetric complex is infinite dimensional, making it impossible to normalize all negative Fourier components of a deformation tensor to zero. The detailed analysis of the 3 -dimensional case is done in [B].

For $n=1$, the symmetric complex assumes the form

$$
0 \rightarrow \Omega_{\sigma}^{(0,0)}\left(H_{(1,0)}\right) \stackrel{\bar{\partial}_{b}}{\longrightarrow} \Omega_{\sigma}^{(0,1)}\left(H_{(1,0)}\right) \rightarrow 0 .
$$

It is convenient to express all tensors in terms of the global framing on $S^{3}$ obtained by restricting the vector fields

$$
Z=z^{\overline{2}} \frac{\partial}{\partial z^{1}}-z^{\overline{1}} \frac{\partial}{\partial z^{2}}, \quad \bar{Z}=z^{2} \frac{\partial}{\partial z^{\overline{1}}}-z^{1} \frac{\partial}{\partial z^{\overline{2}}}, \quad T=-\operatorname{Im}\left(z^{1} \frac{\partial}{\partial z^{1}}+z^{2} \frac{\partial}{\partial z^{2}}\right)
$$

on $\mathbb{C}^{2}$ to $S^{3}$. The dual coframe is the restriction to $S^{3}$ of the 1 -forms

$$
\omega=z^{2} d z^{1}-z^{1} d z^{2}, \quad \bar{\omega}=z^{\overline{2}} d z^{\overline{1}}-z^{\overline{1}} d z^{\overline{2}}, \quad \eta=-\operatorname{Im}\left(\bar{\partial} \log |z|^{2}\right) .
$$

Notice that every deformation tensor is of the form

$$
\phi=\mu \bar{\omega} \otimes Z, \quad|\mu|<1 .
$$

To translate into the coordinates $(w, \theta)$ used above, write $z^{2}=e^{i \theta}\left(1+|w|^{2}\right)^{-1 / 2}$ and $w=z^{1} / z^{2}$ and recall that $\mathrm{e}:=\mathrm{e}_{1}$ is a local framing for $H_{(1,0)}$. A straightforward computation then yields the formulas

$$
\omega=e^{2 \theta i}\left(1+|w|^{2}\right)^{-1} d w \text { and } Z=e^{-2 \theta i}\left(1+|w|^{2}\right) \mathrm{e}
$$

It follows that the tensor $\phi$ has the form

$$
\phi=e^{-4 \theta i} \mu d \bar{w} \otimes \mathrm{e} .
$$

The cohomology of the symmetric complex was computed in [B], where it was demonstrated that

$$
\begin{aligned}
H^{1}\left(\Omega_{\sigma}^{(0, \bullet)}\left(H_{(1,0)}\right), \bar{\partial}_{b}\right) & :=\operatorname{coker} \bar{\partial}_{b} \circ\left(\bar{\partial}_{b}()\right)^{\#} \\
& =\left\{\left(\overline{f_{1}(z)} z^{1}+\overline{f_{2}(z)} z^{2}\right) \bar{\omega} \otimes Z: f_{j}(z) \in \mathcal{C R}\left(S^{3}\right), j=1,2\right\},
\end{aligned}
$$

where $\mathcal{C R}\left(S^{3}\right)$ denotes the space of CR-functions on $S^{3}$-that is, the boundary values of holomorphic functions on the unit ball which are smooth up to the boundary. Thus, by virtue of equation (4), every cohomology class $[\phi] \in$ $H^{1}\left(\Omega_{\sigma}^{(0, \bullet)}\left(H_{(1,0)}\right), \bar{\partial}_{b}\right)$ has a representative with Fourier decomposition of the form

$$
\phi=\sum_{-\infty}^{-4} \phi_{k}
$$

The existence of cohomology classes with representatives having negative Fourier components implies that (at least at the infinitesimal level) there are CR-structures for which it is impossible to find representatives having no negative Fourier components. However the cohomology group $H^{1}\left(\Omega_{\sigma}^{(0, \bullet)}\left(H_{(1,0)}\right), \bar{\partial}_{b}\right)$ is the only obstruction $[\mathrm{B}]$ : 
THEOREM 5.1. Every CR-structure sufficiently near the standard one in the Folland-Stein $s$-norm $(s>6)$ is equivalent, via a small contact diffeomorphism, to one of the form

$$
\phi=\phi_{-}+\phi_{0}+\phi_{+}
$$

where $\phi_{0}=i g d \bar{w} \otimes$ e for the pull back of some real-valued function $g$ on $\mathbb{C} P^{1}$,

$$
\phi_{-}=\left(\bar{f}_{1} z^{1}+\bar{f}_{2} z^{2}\right) \bar{\omega} \otimes Z
$$

for CR functions $f_{j}$ as in (5), and

$$
\phi_{+}=\sum_{k=1}^{\infty} \phi_{k} .
$$

The form (*) is unique up to the choice of a marking on the CR manifold (determined by fixing 7 additional parameters.)

Of these, the only $C R$ structures that embed in $\mathbb{C}^{m}$ for some integer $m$ are those for which $\phi_{-}=0$. Hence, the space of embeddable small deformations of the standard sphere forms a contractible linear space.

Remark. The last part of Theorem 5.1 follows from a stability result of Lempert contained in [L2].

\section{References}

[B] J. Bland, Contact geometry and CR-structures on $S^{3}$, Acta Math. 172 (1994), 1-49.

[BD1] J. Bland and T. Duchamp, Moduli for pointed convex domains, Invent. Math. 104 (1991), 61-112.

[BD2] _, - Basepoint dependence of moduli for convex domains, in preparation (1993).

[BdM] L. Boutet de Monvel, Intégration des équations de Cauchy-Riemann induites formelles, Séminaire Goulaouic-Lions-Schwartz 9 (1974/75), 1-13.

[BE] D. M. Burns and C. L. Epstein, Embeddability for three dimensional CR-manifolds, J. Amer. Math. Soc. 3 (1990), 809-841.

[CL] J. H. Cheng and J. Lee, A local slice theorem for 3-dimensional CR-structures, preprint.

[FS] G. B. Folland and E. M. Stein, Estimates for the $\bar{\partial}_{b}$-complex and analysis on the Heisenberg group, Comm. Pure Appl. Math. 27 (1974), 429-522.

[G] J. Gray, Some global properties of contact structures, Ann. of Math. 69 (1959), 421-450.

[L1] L. Lempert, La métrique de Kobayashi et la représentation des domaines sur la boule, Bull. Soc. Math. France 109 (1981), 427-474.

[L2] - On three dimensional Cauchy-Riemann manifolds, J. Amer. Math. Soc. 5 (1992), 923-969.

[O] H. Omori, Infinite Dimensional Lie Transformation Groups, Lecture Notes in Math. 427, Springer, Berlin, 1994.

[R] H. Rossi, Attaching analytic spaces to an analytic space along a pseudoconvex boundary, in: Proceedings of the Conference on Complex Manifolds, A. Aeppli et al. (ed.), Springer, Berlin, 1965, 242-256.

[W] A. Weinstein, Lectures on Symplectic Manifolds, CBMS Regional Conf. Ser. in Math. 29, Amer. Math. Soc., Providence, 1977. 